\title{
Flow rate measurement in aggressive conductive fluids
}

\author{
Nataliia Dubovikova ${ }^{1, a}$, Yuri Kolesnikov ${ }^{1}$, Christian Karcher ${ }^{1}$ \\ ${ }^{1}$ RTG “Lorentz force velocimetry and eddy current testing", TU Ilmenau, 98693 Ilmenau, Germany
}

\begin{abstract}
Two non-contact experimental methods of flow rate measurements for aggressive conductive liquids are described. The techniques are based on electromagnetic forces and Faraday's law: Lorentz force is induced inside moving conductive liquid under influence of variable magnetic field of permanent magnets. They are mounted along a liquid metal channel or (in case of the second method) inserted into rotated metal wheels. The force acts in the opposite of fluids' velocity direction and hence it is possible to measure reaction force of it that takes place according to Newton's law on magnetic field source - permanent magnets. And by knowing the force, which linearly depends on velocity, one can calculate mean flow rate of liquid. In addition experimental "dry" calibration and its results are described for one of the measurements' techniques.
\end{abstract}

\section{Introduction}

In many continuous metallurgical processes like production of secondary aluminium [1] or the casting of steel [2] the knowledge of flow rate is crucial for successful process control and thus for the quality of the product. However, due to the chemical aggressive of liquid melts at high temperature, only non-contact measurement techniques can be employed. Since metal melts are excellent electrical conductors, the employment of electromagnetic flow measurement devices are favourable, see [3] for an overview of such devises. One of these methods is Lorentz force velocimetry (LFV) [4] that can be used for both volumetric flow rate measurements [5] and local velocity measurements [6]. This method has been invented and patented at Technische Universität Ilmenau [7].

Lorentz force velocimetry is based on the fundamental principles of magentohydro-dynamics (MHD) [8], [9], [10]. When an electrically conducting material passes the field lines stretched by an arrangement of permanent magnets, eddy currents are induced inside the moving material. These eddy currents interact with the applied magnetic field and, as a consequence, Lorentz forces are generated within the material. In more detail, this process can be divided into three parts:

(i) the movement of a metal melt in an externally applied static magnetic field causes an e.m.f. (Faraday's law of induction) and electrical eddy currents are generated;

(ii) the generated eddy currents induce a secondary magnetic field (Ampere's law) that adds to the applied primary magnetic field; (iii) the combined magnetic field interacts with induced current to give rise to Lorentz forces. This forces act within the metal melt tending to inhibit the melt movement, i.e. breaking the flow. In continuous casting of steel this effect is used to dissipate the kinetic energy of liquid metal jet entering from the tundish into the mould via the submerged entry nozzle [11].

In the present case we employ these principles the other any around, i.e. to pump the melt by Lorentz forces generated within the melt due to the rotation of permanent magnets. Moreover, since at constant temperature and thus constant melt properties, Lorentz force linearly depends on flow rate only, upon measuring the force, flow rate can be evaluated.

In the paper we present two methods to measure this force: directly by cross-correlation function of two strain gauge force sensors and indirectly by recoding the corresponding torque acting on the shaft of the pump using a digital torque sensor. Thus, in the second case flow driving and flow measurement can be combined using a single device. Due to indirect measurement, calibration of the sensor is necessary in order to determine the specific lever of the device.

\section{Theoretical backgrounds}

In the following we give a short summary of the theoretical background that forms the basis of the present measurement principle. As is well known from liquid metal MHD, characterized by relatively small magnetic Reynolds numbers [12], Ohm's law for a moving liquid can be written in the following form:

$$
\mathbf{j}=\sigma\left(-\nabla \Phi+\left(\mathbf{u} \times \mathbf{B}_{\mathbf{0}}\right)\right)
$$

\footnotetext{
a Corresponding author: nataliia.dubovikova@tu-ilmenau.de
} 
where $\sigma$ is the electrical conductivity, $\mathbf{j}$ denotes electric current density, $\Phi$ is the electric scalar potential, $\boldsymbol{v}$ is the melt velocity, and $\mathbf{B}_{0}$ denotes the magnetic field. Ohm's expresses the fact that in an electrically conducting liquid, eddy currents are induced by the movement of the liquid through a magnetic field and the action of a curlfree electrical field, defined by the gradient of its scalar potential.

Due to the solenoidal nature of $\mathbf{B}_{0}$ and charge conservation we have the following constitutional equations,

$$
\begin{gathered}
\nabla \cdot \mathbf{B}_{\mathbf{0}}=0, \\
\nabla \cdot \mathbf{j}=0, \\
\nabla^{2} \Phi=\mathbf{B}_{\mathbf{0}} \cdot(\nabla \times \mathbf{u}) .
\end{gathered}
$$

The mutual interaction of the eddy currents and the applied magnetic field generates a Lorentz force density $f$ defined by the relation

$$
\mathrm{f}=\mathbf{J} \times \mathbf{B}_{\mathbf{0}} .
$$

We now insert the characteristic values $v, B_{0}$, and $L$ for velocity, magnetic induction, and length, respectively, into Eq. (1)-(5). Upon integrating over the volume affected by the magnetic field we find the following important scaling relations for the induced Lorentz force.

$$
\begin{aligned}
& F_{L} \propto \sigma v B_{0}^{2} L^{3} \\
& F_{L} \propto \sigma Q B_{0}^{2} L,
\end{aligned}
$$

where $Q$ is the volumetric flow rate. According to Eq. (7), Lorentz force linearly depends on the product of electrical conductivity and flow rate.

\section{Experimental techniques}

During engineering problems investigations both types of scientific methods - experimental and analytical should be used. They supplement each other and are part of the world research process. Main advantages of experimental methods are reproducibility, selectivity and possibility to create artificially any conditions, even extreme mode (allows one to observe the object in extreme conditions).

Within this chapter two experimental methods of flow rate determination for conductive fluids are described. Elements of measurement techniques that are based on the LFV are shown on the example of created in TU Ilmenau closed channel with Galinstan - alloy of gallium, indium, and stannum (Latin for "tin"), which is liquid under room temperature. Two types of measurement systems, that are mounted to the channel, are described below.

\subsection{Time-of-flight LFV}

The method time-of-flight LFV [4] is intended for contactless determination of flow rate in conductive fluids. It can be successfully used even in case when such material properties as electrical conductivity or density are not precisely known under specific outer conditions. The last reason makes time-of-flight LFV especially important for industry application.

According to time-of-flight LFV (figure 1) two coherent measurement systems are mounted on a channel one by one. The measurement is based on getting of cross-correlating function of signals, which are registered by two magnetic measurement's system. Every system consists of permanent magnet and force sensor, so inducing of Lorentz force and measurement of the reaction force are made simultaneously.

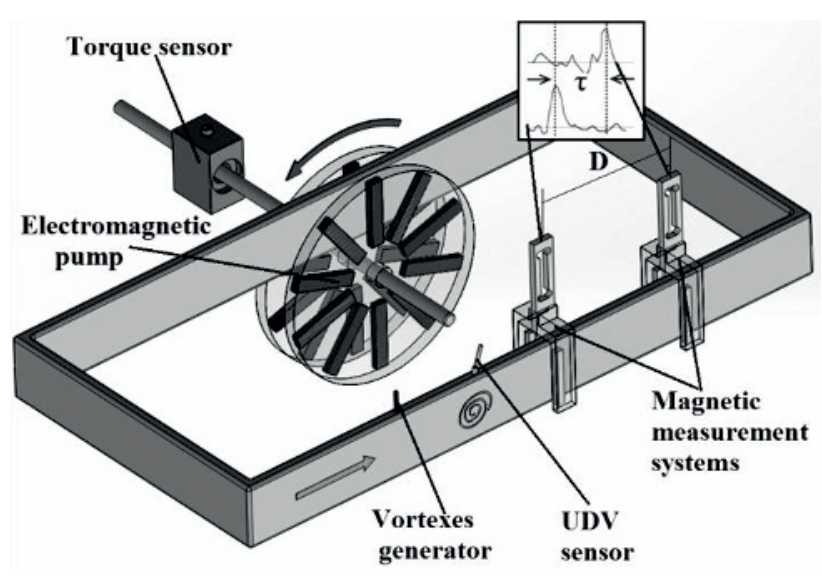

Figure 1. Experimental setup for LFV and closed channel with liquid metal (Galinstan). Three measurement devices are mounted here for flow rate determination: e/m pump, UDVsensor, time-of flight Lorentz force flowmeter

Any cross-correlation function is useful only in case of qualitative difference between signals and for creating the difference in this case turbulent fluctuations are used. Before reaching of measurement zone of a channel liquid passes artificial vortex generator that induces strong disturbances in it. Reynolds number inside the channel is proportional to $10^{4}$, so Karman's street is forming after artificially created barrier. And vortexes, which are generated within the Karman's street, provide changing in velocity profiles for every magnetic measurement system.

A generator of vortexes is located in such way, that vortex can move with a liquid through magnetic field of permanent magnets. And when such fluctuation reaches magnetic field of measurement system we can observe a peak on its force-time characteristic while second system still measures stable flow. Then, according to the time between peaks and the distance between measurement systems, observer can estimate mean velocity and, hence, flow rate of the liquid. Thus, by knowing a distance between magnet systems $\mathrm{D}$ and time between peaks $\tau$ one can calculate mean velocity by velocity definition (distance D per time $\tau$ ) and then by multiplying it with experimental coefficient $k_{L}$ (coefficient depends on geometry of a channel and liquid's characteristics) it is possible to obtain the value of liquid's volumetric flow rate.

$$
Q_{v o l}=k_{L} \cdot D / \tau
$$


Vortexes inside the experimental channel can be generated by using a solid rod, which is inserted into a channel, but this method can be put into practice only in laboratory conditions. At industry application contact vortex generator can't be applied - temperature of investigated liquids there can reach thousand kelvins. For this reason non-contact method of vortexes generation can be used: for example, doubled permanent magnets, system of conductors under influence of direct current. Such facilities can be mounted from both sides of the defined area of channel's wall and thus create magnetic obstacles [13] inside a flow by magnetic field influence.

\subsection{LFV by torque measurement}

Another method of LFV measurement includes applying of electromagnetic pump as flow-controlling and as flowmeasurement device at the same time.

Figure 2 shows the principle scheme of such a pump. The pump basically consists of a pair of metal disks, on each of which a total of 20 finger-type permanent magnets are amounted. The disks are arranged on a joining shaft that is connected to an electrical motor. By controlling motor power, we can control rotation frequency $\mathrm{n}$ of the shaft. A digital strain gage torque sensor is mounted on the shaft to measure the Lorentz force that is exerted on an electrical conductor placed within the gap between the two rotating disks.

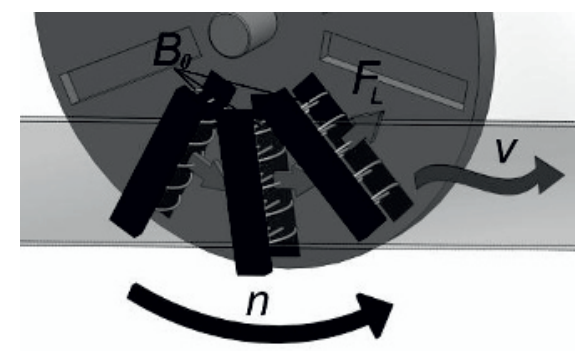

Figure 2: The principal scheme of EM pump on the basis of rotating permanent magnets. Lorentz force induced by relative motion of magnetic field and conductive fluid and pushes liquid metal inside the channel between disks of the pump.

Using such an arrangement the melt flow rate $Q$ can be estimated by evaluating the following equation.

$$
Q=k T /\left(\sigma B_{0}^{2} L l\right)
$$

where $T$ is the measured torque, $l$ its respective lever, and $k$ is a device factor. This factor depends on a number of specific experimental parameters like the aspect ratio of the flow channel, the actual geometric arrangement of the permanent magnets, among others. Hence, the specific value $k$ of has to be obtained individually for every experimental setup.

Standard strain gauge torque sensor is used for torque measurement within the method. The sensor is mounted on the pump's shaft and the measured torque is proportional to liquid metal's velocity.

As is obvious from Eq. (9), to evaluate the flow rate knowledge of the torque lever $l$ is necessary. For obtaining of specific value of $l$ value one needs to perform calibration procedure, i.e. measuring Lorentz force $F_{L}$ and torque $T$ for various rotation frequencies in order to find the functional relation

$$
l=[T(n) /(F(n)] .
$$

However, calibration of the device using liquid metal is complicated, time-consuming, and expensive. Therefore we perform a so-called dry calibration procedure. In this case the lever becomes independent of the rotation frequency. The main idea of dry calibration is to model the liquid by a solid electrically conducting nonmagnetic material like solid aluminium bars or sheets. As the electrical conductivity of a metal in the solid state is about one order of magnitude greater than in the solid state, a proper scaling scheme has to be employed in order to extrapolate the data to the regime of melt flow.

The dry calibration procedure can be carried out as it is shown on figure 3 .

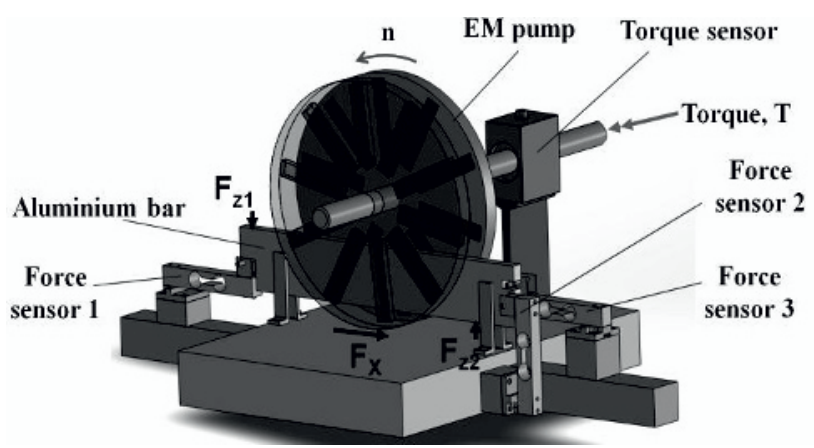

Figure 3. Experimental set-up used in the dry calibration procedure. Lorentz force pushes the aluminium sheet arranged between the disks of the pump and two components of the force are measured by force sensors 1, 2, 3

Figure 3 illustrates the experimental set-up for the dry calibration procedure, at that $\mathrm{F}_{\mathrm{z} 1}, \mathrm{~F}_{\mathrm{z} 2}, \mathrm{~F}_{\mathrm{x}}$ are vertical (z) and horizontal (x) components of Lorentz force $F_{L}$ respectively. During the calibration we place aluminium sheets of various dimensions in the gap between the rotating disks. Upon varying the rotation frequency of the disks we record the Lorentz forces acting on the sheet by using a total of 3 strain gage force sensors. In more detail, sensors 1 and 3, arranged at both ends of the long side of the sheet, measure the respective $z$-components of Lorentz force, $F_{z 1}$ and $F_{z 2}$, i.e. the electromagnetic lift force that adds to the gravitational force of the sheet, see Fig. 2. On the other hand sensor 2 measures the $x$ component of the Lorentz force, $F_{x}$, i.e. the electromagnetically induced drag force. As the sheets are positioned exactly symmetrically in the gap between the disks, no significant y-component of Lorentz force arises. Finally, the strain gage torque sensor measures the overall torque acting on the pump shaft. Strain gage sensors were chosen because of their long-term stability with static loads and high-precision characteristics. Eventually the overall force contributing to the torque can be calculated by geometrical superposition of the respective component. As a result we can evaluate the lever for each sheet used in the calibration procedure. In our investigations we apply a total of three different sheets of thicknesses $5 \mathrm{~mm}, 10 \mathrm{~mm}$, and $15 \mathrm{~mm}$. The 
height and the length of the sheets are fixed at $30 \mathrm{~mm}$ and $600 \mathrm{~mm}$, respectively.

Asynchronous AC motor with maximal rotation frequency 945 r.p.m. and power $2.2 \mathrm{~kW}$ was used as a driver for pump's rotation.

Certainly, proposed way of calibration of LFV by torque measurement device can solve only one significant problem - determination of the lever for specific geometry of a channel. For finding of experimental coefficient $j$ one should perform fluid experiments with the liquid, which is planned to be used during main experiment within a channel.

\subsection{Results of pump's calibration}

Our main findings are summarized in figures 4 and 5 . In figure 4 we show the overall Lorentz force, measured by the three digital force sensors, as a function of the rotation frequency for various thicknesses of the sheet. Figure 5 shows the resulting torque acting on the shaft as measured by the digital torque sensor. In both graphs we use a scaled representation of the measured values. The re-scaling has been performed to eliminate the inherent dependence of the data on the width of the sheet. In detail we use the scaling:

$$
\begin{aligned}
F_{S C}= & F_{L}(H / W) \\
& \text { and } \\
T_{S C}= & T(H / W),
\end{aligned}
$$

where $H$ and $W$ denote the distance between wheels and the width of the sheet, respectively. The motivation for this re-scaling stems is explained below.

\section{Scaled Lorentz force $F s c, \mathbf{N}$}

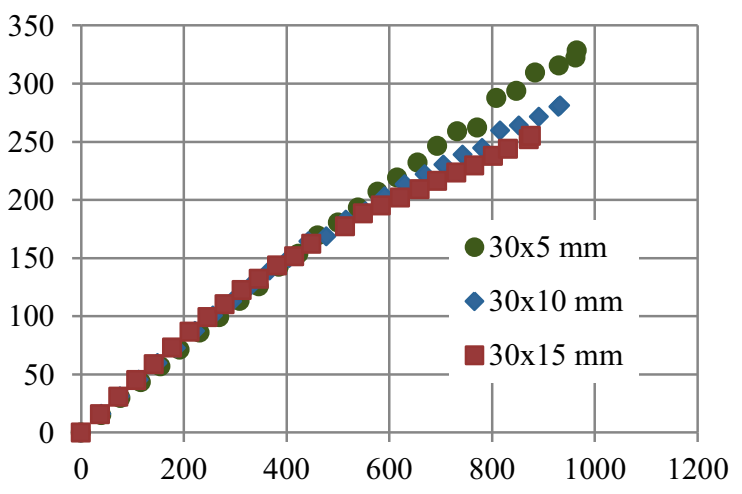

Rotation frequency $n$, r.p.m.

Figure 4. Measured force for different plate's dimensions under variable rotation frequency of the pump. A component of equation $I=\left[T_{s c} / F_{S c}\right]$ for calculating of lever's value

As a rule Lorentz force and produced by it torque increase with increasing of cross-section of the conductive material. It causes by several different reasons that include:

(i) width of an aluminium plate. With a change of a plate's width as a consequence two parameters are changing: volume of a conductive material that is influenced by magnetic field, and its electric resistance to induced eddy current. In thicker plates the induced eddy current experiences less electric resistance as it has more space for an easy looping back in given volume. Besides, magnetic field distribution in between of wheels is not homogeneous: it decreases from walls to the middle of gap. Hence magnetic field, which passes through thick plate has higher value than through thin one.

(ii) width of the gap between wheels of e/m pump. With an increasing of gap's width value of magnetic field in the middle is decreasing due to change of distance between permanent magnets.

(iii) change of magnetic Reynolds number $R e_{m}$. An increase of width results in an increase of the magnetic Reynolds number $R e_{m}$ given by the relation by $R e_{m}=$ $\mu \sigma u L$, where $\mu$ is the magnetic field constant. This increase is due to the increase of the electromagnetic interaction length $L$. As it is known from MHD, larger values of $R e_{m}$ result in stronger induced magnetic fields that fight against the applied primary field [12]. This give rise to a weakening of the overall magnetic field that contributes to the Lorentz force. Therefore lower values for both $F_{L}$ and $T$ are observed in cases of thicker plates.

Scaled torque $T s c, \mathrm{~N} \cdot \mathrm{m}$

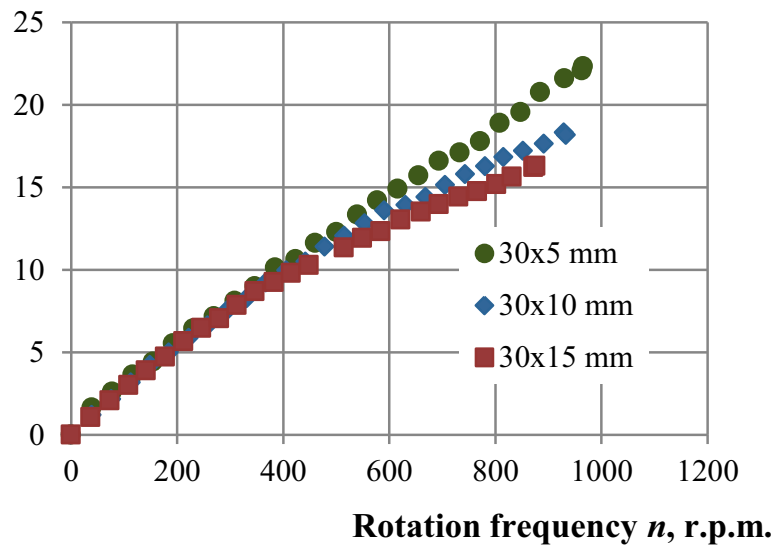

Figure 5. Measured torque for different plate's dimensions under variable rotation frequency of the pump. A component of equation $I=\left[T_{S C} / F_{S C}\right]$ for calculating of lever's value

Influence of first two effects can be easily observed on the graphs: after re-scaling of Lorentz force and torque by the width factor (Eq. (11) and (12)) there are no difference between curves on the low rotation frequency level. $R e_{m}$ increases its influence on the results with higher values of rotation frequency. It can be seen on the graphs as perceptible discrepancy of the curves after rotation frequency about 500 r.p.m. Measured Lorentz force and torque after this point have noticeably lower values for thicker plate because in this case induced by Lorentz force magnetic field becomes the same order as applied by permanent magnets magnetic field. And though they have different directions, the resulting magnetic field is decreasing as well as induced Lorentz force.

Measurements results are presented for three investigated dimensions of aluminium plates (figure 6). Mean value of obtained lever $l$ for three dimensions of the plates is equal to $0.067 \mathrm{~m}$. The difference between 
values according to thickness of plate is occurred due to thermal effect that caused by induction heating. Eddy currents can significantly increase value of temperature of plate and the thinner is the plate - the higher temperature's value it can reach.

The obtained value $l$ is less than the distance from shaft's middle to magnets' centre. It takes place because in case of round pump we have several magnets, which influence on the plate and only part of them are acting perpendicularly to it. All other inserted magnets have different levels of inclination, and i.e. their affected zone is higher than middle line of the plate.

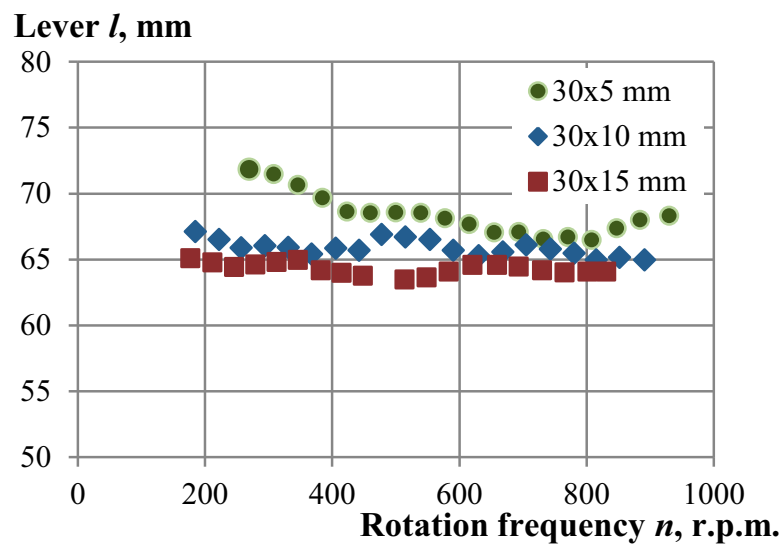

Figure 6. Lever $l$ of measured torque $T$ on the shaft of e/m pump due to induced Lorentz force $F_{L}$ in aluminium plate under different rotation frequencies $n$

\section{Summaries}

Time-of-flight LFV and LFV by torque measurement are very prospective techniques for aggressive liquid's mean velocity and volumetric flow rate measurements, especially in relation to its industry applications. Our investigation shows that proposed methods of flow rate measurement are prospective techniques in row of other contactless methods, which can be applied in cases of high temperature liquid metal examination. Feasibility of the method to determine flow parameters without probes and signal particles is significant under condition of industrial manufacture, where contact ways of velocity and flow rate measurements are not acceptable. At the time pre-calibration and preliminary experiments with the prototype are continuing and during them the facilities have shown stable and reproducible results. According to obtained data significant factors that are influenced on measured Lorentz force, were deeply investigated.

\section{Acknowledgements}

We would like to express appreciation to DFG for funding of our Research Training Group "Lorentz force velocimetry and Lorentz force eddy current testing" and this research particularly. Our thanks are extended to Prof. Thess for his useful and constructive scientific discussions and to Jonas Ketterer for his support in measurements.

\section{References}

1. Y. Kolesnikov, Ch. Karcher, A. Thess, Metallurgical and Materials Transactions B, 42B (2011).

2. Ch. Karcher Y. Kolesnikov and A. Thess, Proc. TMS Annu. Meeting (Seattle, WA, USA 2010).

3. J. Priede, D. Buchenau and G. Gerbeth: J. Appl. Phys. 110 (2011).

4. A. Thess, E. Votyakov, B. Knaepen, O. Zikanov, New Journal of Physics, 9 (2007).

5. D. Jian, Ch. Karcher, Meas. Sci. Technol. 237 (2012).

6. A. Thess, Y. Kolesnikov and Ch. Karcher 2007 Patent WO2007/033982

7. C. Heinicke, S. Tympel, G. Pulugundla, I. Rahneberg, T. Boeck, \& A. Thess, Journal of Applied Physics, 112 (2012).

8. P. A. Davidson An Introduction to Magnetohydrodynamics (Cambridge: Cambridge University Press, 2001).

9. R. Moreau Magnetohydrodynamics (Dordrecht: Kluwer 1990).

10. J.A. Shercliff The theory of electromagnetic flowmeasurement (Cambridge: Cambridge University Press, 1962).

11. K. Cukierski and B.G. Thomas, Metallurgical and Materials Transactions B, Vol. 39, Nr. 1, (2008).

12. H. Keith Moffatt, Reflections on Magnetohydrodynamics. (ISBN 0-521-53169-1) (Ed. G.K. Batchelor, H.K. Moffatt \& M.G. Worster) Cambridge University Press, (2000).

13. Y. Kolesnikov, A. Thess, Advances in Turbulence XII, Springer proceedings in Physics, 132 (2009). 